\title{
Multi-Planar Deep Segmentation Networks for Cardiac Substructures from MRI and CT
}

\author{
Aliasghar Mortazi ${ }^{1}$, Jeremy Burt ${ }^{2}$, Ulas Bagci ${ }^{1}$ \\ ${ }^{1}$ Center for Research in Computer Vision (CRCV), University of Central Florida, \\ Orlando, FL. \\ 2 Diagnostic Radiology Department, Florida Hospital, Orlando, FL. \\ E-mail: a.mortazi@knights.ucf.edu
}

\begin{abstract}
Non-invasive detection of cardiovascular disorders from radiology scans requires quantitative image analysis of the heart and its substructures. There are well-established measurements that radiologists use for diseases assessment such as ejection fraction, volume of four chambers, and myocardium mass. These measurements are derived as outcomes of precise segmentation of the heart and its substructures. The aim of this paper is to provide such measurements through an accurate image segmentation algorithm that automatically delineates seven substructures of the heart from MRI and/or CT scans. Our proposed method is based on multi-planar deep convolutional neural networks (CNN) with an adaptive fusion strategy where we automatically utilize complementary information from different planes of the 3D scans for improved delineations. For CT and MRI, we have separately designed three CNNs (the same architectural configuration) for three planes, and have trained the networks from scratch for voxel-wise labeling for the following cardiac structures: myocardium of left ventricle (Myo), left atrium (LA), left ventricle (LV), right atrium (RA), right ventricle (RV), ascending aorta (Ao), and main pulmonary artery (PA). We have evaluated the proposed method with 4-fold-cross-validation on the multi-modality whole heart segmentation challenge (MM-WHS 2017) dataset. The precision and dice index of 0.93 and 0.90 , and 0.87 and 0.85 were achieved for $\mathrm{CT}$ and MR images, respectively. While a CT volume was segmented about 50 seconds, an MRI scan was segmented around 17 seconds with the GPUs/CUDA implementation.
\end{abstract}

Keywords: cardiovascular disorders, computed tomography, cardiac magnetic resonance imaging, convolutional neural network, whole heart segmentation

\section{Introduction}

According to the World Health Organization [1, cardiovascular diseases (CVDs) are the first cause of death globally. About 17.7 million people died from CVDs in 2015, which was $31 \%$ of total global deaths from diseases. Almost 7.4 million of these deaths were due to CVDs and about 6.7 million were due to the stroke. 
Extensive research and clinical applications have shown that both CT and MRI have vital roles in non-invasive assessment of CVDs. CT is used more frequently than MRI due to its fast acquisition and cheaper cost. On the other hand, MRI has an excellent soft tissue contrast and no ionizing radiation. However, most commercially available image analysis methods have been either tuned for CT or MRI only. Furthermore, many studies are focused on only one substructure of the heart (for instance, the left ventricle or left atrium). Surprisingly, there is very little published research on segmenting all substructures of the heart despite the fact that clinically established markers rely on shape, volumetric, and tissue characterization of all the cardiac substructures. Our study is concerned with this open problem from a machine learning perspective. We have investigated architectural designs of deep learning networks to solve multi-label and multimodality image segmentation challenges within the scope of a limited GPU and imaging data.

Related Works. Literature related to cardiac image segmentation is vast. Among these works, atlas-based methods have been quite popular and favored for many years. For instance, multi-atlas based whole-heart segmentation using MRI and CT by [2] and atlas propagation based method using prior information by [3] are a few key examples. Despite their accuracy, those methods often lack efficiency due to heavy computations on the registration algorithms (e.g., from 13 minutes to 11 hours of computations reported in the literature). Interested readers can find a survey paper on cardiac image segmentation methods in [4] for a full list of methods and their comparative evaluations.

More recently, deep learning based approaches are replacing the conventional methods in medical image segmentation fields in general, and cardiac field in particular. For instance, in [5], a multi-planar deep learning has been utilized to segment LA and pulmonary veins from MR images. A recurrent fully convolutional neural network has been proposed to segment LV from MRI in 6]. In a similar fashion, a deep learning algorithm combined with a deformable-model approach was used to segment LV from MRI [7]. In [8, RV segmentation has been accomplished through a joint localization and segmentation algorithm within a deep learning framework. To date, the majority of deep learning methods have segmented only one or two structures of the heart and constrained to only one modality, unlike what is presented herein.

Our Contributions. We have constructed a network structure similar to the one devised in [5], which segments the left atrium and proximal veins from MRI successfully. In this paper, we have extended this segmentation engine in several different ways as follows. (1) A deeper CNN has been utilized as compared to [5. (2) We have used both CT and MRI to test and evaluate the proposed system while Mortazi et al. used only MRI [5]. (3) We have extended the binary segmentation problem into a multi-label segmentation problem. (4) We have devised a rank based adaptive fusion method to assess effective information from 


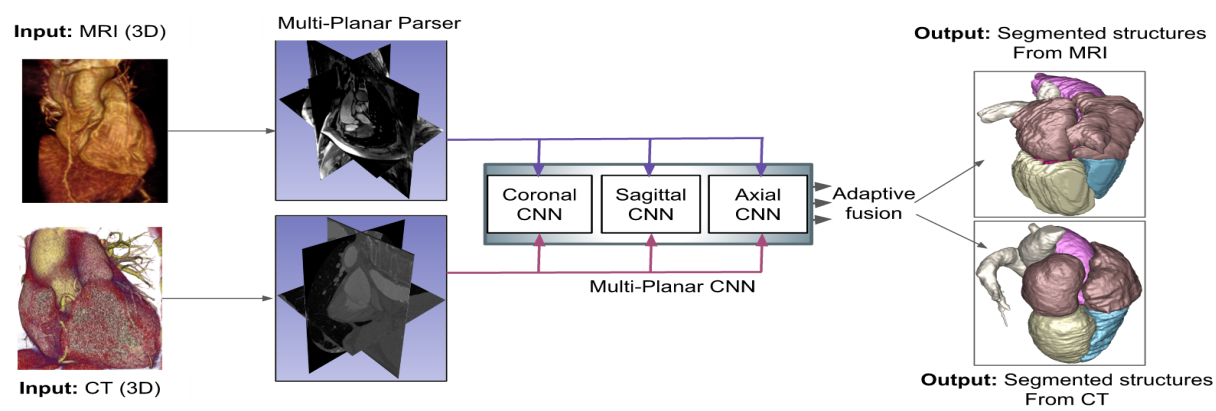

Fig. 1. Overall view of Mo-MP-CNN with adaptive fusion

different planes for all delineated objects and select the best fusion strategies for highly accurate and efficient delineation results.

\section{Multi-Object Multi-Planar CNN (MO-MP-CNN)}

The proposed method is called multi-object multi-planar convolutional neural networks (MO-MP-CNN), and its modules are illustrated in Figure1. MO-MPCNN takes 3D CT or MR scans as an input and parses it to three perpendicular planes: Axial(A), Coronal(C), and Sagittal(S). For each plane (and modality), a 2D CNN is trained to label pixels. CNNs have been trained from scratch to adapt into CT and MRI context. After training each of the 2D CNNs separately, adaptive fusion strategy is utilized by combining the probability maps of each of the CNNs. The details of the CNN and adaptive fusion method are explained in the following.

CNN network. The proposed encoderdecoder based network architecture is illustrated in Fig.2. Twelve convolution layers have been used in encoder and decoder separately. In the encoder part, two max-pooling layers have been used to reduce the dimension of the image by half and in decoder part two upsampling layers (bilinear interpolation) have been used to get the image back to its original size. The size of all filters were set as $3 \times 3$. Each convolution layer is followed by a batch normalization and Rectified Linear Unit $(R e L U)$ as an activation function. The number of filters in the last convolu-

Table 1. Data augmentation parameters.

\begin{tabular}{|c|c|c|}
\hline \multicolumn{3}{|c|}{ Data augmentation } \\
\hline Methods & \multicolumn{2}{|c|}{ parameters } \\
\hline Zoom in & Scale $\varepsilon[1.1,1.3]$ \\
\hline Rotation & $k \times 45, k \varepsilon[-1,1]$ \\
\hline \multicolumn{2}{|c|}{ Training images $(\boldsymbol{C T})$} \\
\hline CNN & \# of images & Image size \\
\hline Sagittal & 40,960 & $350 \times 350$ \\
Axial & 21,417 & $350 \times 350$ \\
Coronal & 40,960 & $350 \times 350$ \\
\hline \multicolumn{2}{|c|}{ Training images } & $($ MRI $)$ \\
\hline CNN & \# of images & Image Size \\
\hline Sagittal & 20,074 & $288 \times 288$ \\
Axial & 29,860 & $288 \times 160$ \\
Coronal & 19,404 & $288 \times 288$ \\
\hline
\end{tabular}
tion layer is equal to the number of 
classes (i.e., 8 (background +7 objects)) and is followed by a softmax function to make a final probability map for each object. Similar to [5], the simplified z-loss 9] function has been used to train the network. To provide a sufficient number of training images for the networks, data augmentation has been applied to the training images by rotation and zoom-in operations. The details of the augmentation and the number of data for each CNN are summarized in Table 1.

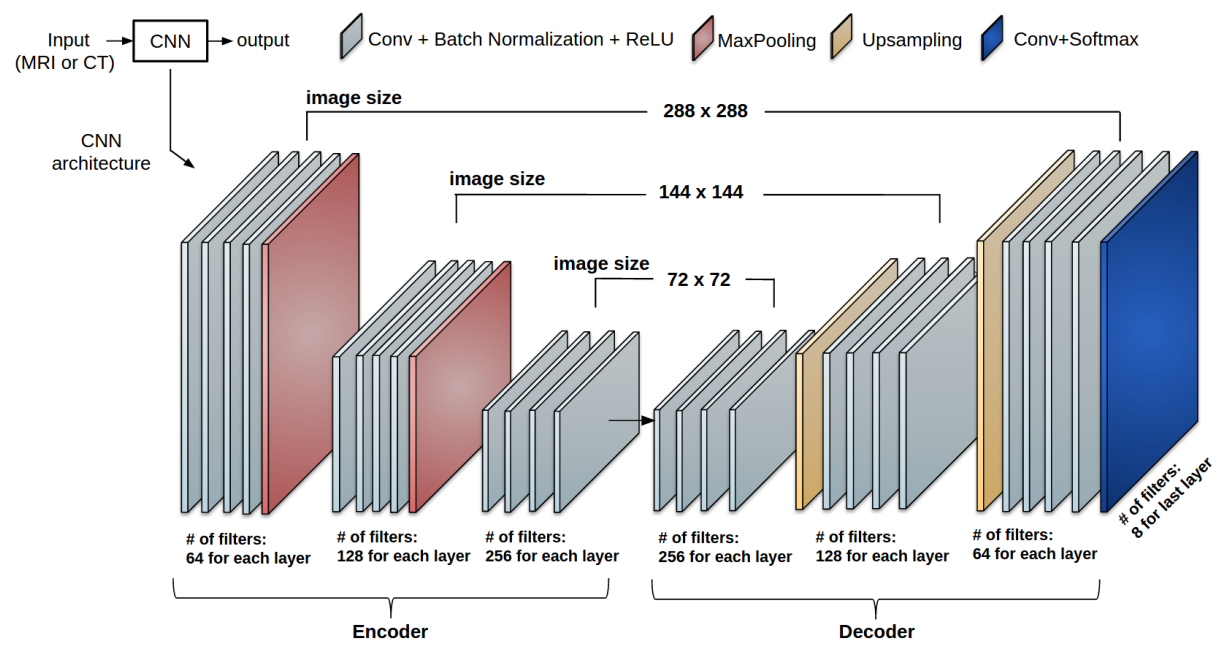

Fig. 2. Details of the CNN architecture. Note that image size is not necessarily fixed for each plane's CNN.

Multi-object adaptive fusion. An adaptive fusion strategy has been extended in the way that it can be applied to multi-object segmentation instead of binary segmentation. Let $\mathbf{I}$ and $\mathbf{P}$ denote an input and output image pair, where output is the probability map of the CNN. Also, let the final segmentation be denoted as o. As shown in Fig, 3 , o is obtained from the probability map $\mathbf{P}$ by taking the maximum probability of each pixel in all classes (labels). Then, a connected component analysis (CCA) is applied to o to select reliable and unreliable regions, where unreliable regions are considered to come from false positive findings. Although this approach gives a "rough" estimation of the object, this information can well be used for assessing the quality of segmentations from different planes. If it is assumed that $\mathbf{n}$ is the number of classes (structures) in the images and $\mathbf{m}$ is the number of components in each class, then connected component analysis can be performed as follows: $C C A(\mathbf{o})=\left\{o_{11}, \ldots, o_{n m} \mid \cup o_{i j}=\mathbf{o}\right.$, and $\left.o_{11}, \ldots, o_{n m} \mid \cap o_{i j}=\phi\right\}$. For each class $\mathbf{n}$, we can now assign reliability parameters (weights) to increase the influence of planes that have more reliable (trusted) segmentations as follows: $w=\sum_{i}\left\{\max _{j}\left\{\left|o_{j}\right|\right\}\right\} / \sum_{i j}\left|o_{i j}\right|$, where $w$ indicates a weight parameter. In our 
interpretation of the CCA, the difference between trusted and non-trusted regions have been used to guide the reliability of the segmentation process: the higher the difference is, the more reliable the segmentation is (See Fig.3, weight distribution w.r.t the difference). In test phase, we have simply used those predetermined weights from the training stage.

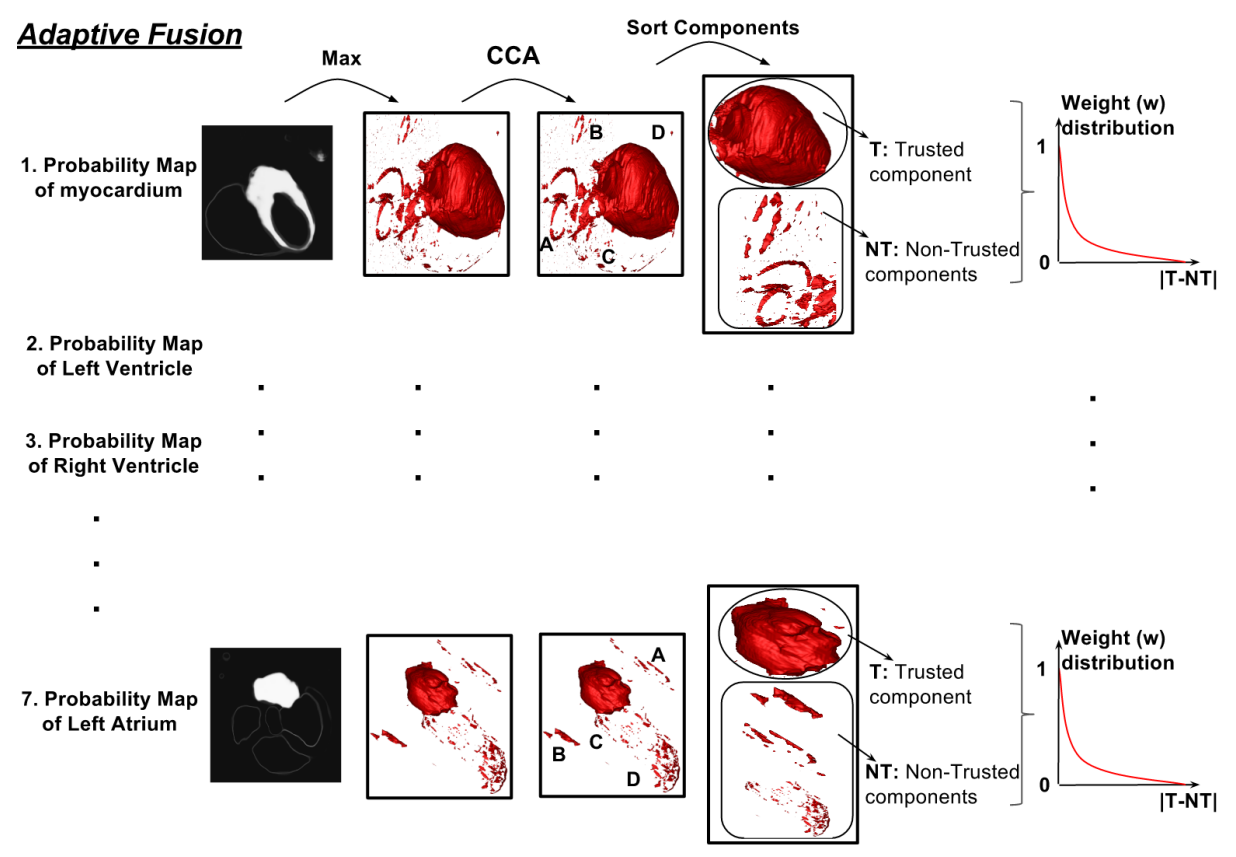

Fig. 3. Connected components obtained from each plane were computed and the residual volume (T-NT) was used to determine the strength for fusion with the other planes.

\section{Experimental Results}

Dataset and preprocessing: For the experiments and evaluations of the proposed method, we used the STACOM 2017 for whole heart segmentation challenge dataset, containing $20 \mathrm{MR}$ and $20 \mathrm{CT}$ images for training (with groundtruth) and 40 test images without ground-truth for each modality. We performed a 4 fold cross-validation on the dataset such that 15 subjects were used for training and 5 subjects have been chosen for validation for each fold. The CT images were obtained from routine cardiac CT angiography and to cover the whole heart, extending from the upper abdomen to the aortic arch. Axial in-plane resolution was $0.78 \times 0.78 \mathrm{~mm}$ and slice thickness was $1.6 \mathrm{~mm}$. The MR images were acquired by using $3 \mathrm{D}$ balanced steady state free precession (b-SSFP) sequences, with about $2 \mathrm{~mm}$ acquisition resolution in each direction. In preprocessing step, anisotropic smoothing filtering was applied to both CT and MR images prior to segmentation. In addition, histogram matching was used for MR images to alleviate intensity non-standardness issues. 
$6 \quad$ Aliasghar Mortazi, Jeremy Burt, Ulas Bagci

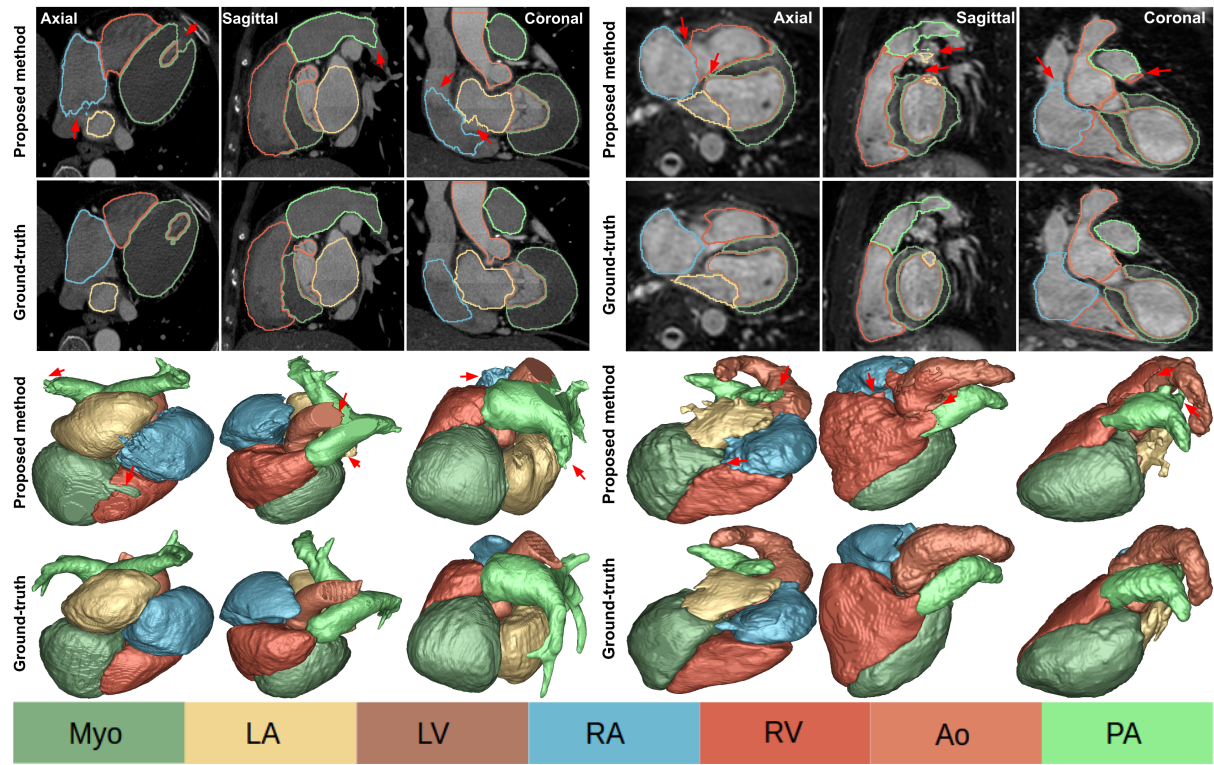

Fig. 4. First two rows show axial, sagittal, and coronal planes of the CT (first three columns) and MR images (last three columns), annotated cardiac structures, and their corresponding surface renditions (last two rows). Red arrows indicate some of missegmentations.

Table 2. Quantitative evaluations of the proposed segmentation method for both CT and MRI are summarized.

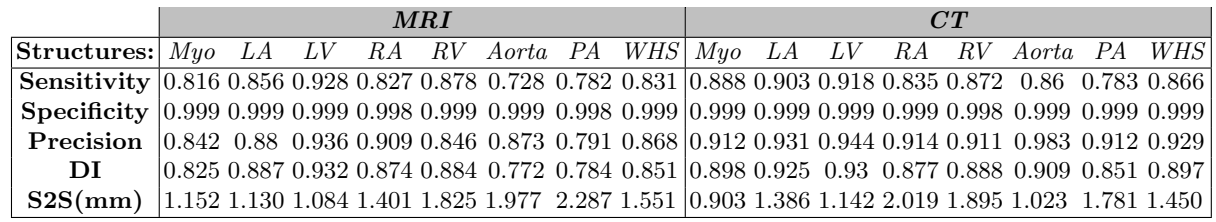

Evaluation: Five metrics were assessed: sensitivity, specificity, precision, dice index (DI), and surface to surface (S2S) distance. A summary of the findings for each structure and also for the whole heart are reported in Table 1. The WHS is the average of all structures. The box-plot for sensitivity, precision, and DI for both CT and MRI and for all structures are shown in Fig. 5 . The qualitative results (including difficult cases for segmentation) for CT and MR modalities are illustrated in Fig. 4. Algorithms were implemented on the Nvidia TitanXp GPUs using Tensorflow [10. The average time for segmenting the whole heart from the CT volume using three TitanXp GPUs was about 50 seconds. Segmenting using MR volume took about 17 seconds. For comparison, the time on the Intel Xeon Processor E5-2620 with 8 cores for CT images was about 30 minutes and for MR images was about 8 minutes. 


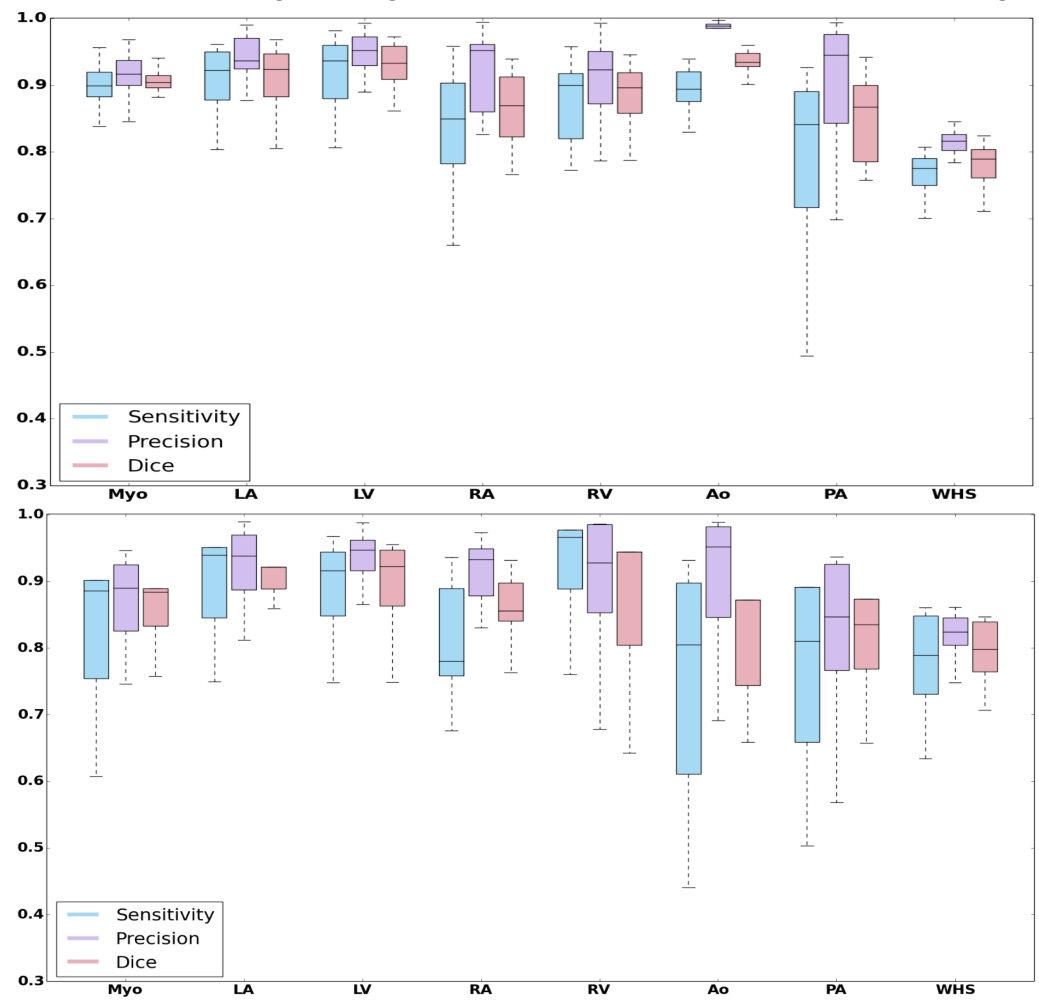

Fig. 5. Box plots for sensitivity, precision, and Dice index for each structure and WHS. Top figure is for CT dataset and bottom figure is for MR dataset

\section{Discussion and Conclusion}

The main goal of the current study is to develop a framework for accurately segmenting the all cardiac substructures from both CT and MR images with high efficiency. The main strength of the proposed method is to train multiple CNNs from scratch and to allow an adaptive fusion strategy for information maximization in pixel labeling despite the limited data and hardware support. Our findings indicate that MO-MP-CNN can be used as an efficient tool to delineate cardiac structures with high precision, accuracy, and efficiency.

Technically, one may question why we did not employ a completely 3D CNN approach instead of utilizing a multi-planar fusion of multiple 2D CNNs. As discussed in [5], the lack of a large number of 3D images restricts the depth of CNN training, which may highly likely result in sub-optimal implementation. Hence, training large number of $2 \mathrm{D}$ slices is much more feasible than utilizing $3 \mathrm{D}$ approach with the current setting. In the instance of plentiful GPU processing power and 3D imaging data, training would be optimized using a 3D CNN.

Another limitation of our work stems from the use of the softmax function in the last layer of the proposed network. To explore whether the information loss due to class normalization in this step is significant, further research should 
be undertaken using information from the layer before the softmax in fusion part and compared with the current system. Finally, further work is needed to establish comparative evaluation of different deep neural network approaches such as ResNet, U-net, and others. While deeper networks are desirable to achieve higher precision in segmentation tasks, lack of 3D data is a significant limitation for training such a system. Data augmentation and transfer learning have been shown to adequately address such challenges to a certain degree, but there is currently no research proving the optimality of such networks relative to the availability of data at hand.

\section{References}

1. "Cardiovascular Diseases (cvds)," http://www.who.int/mediacentre/ factsheets/fs317/en/, 2007, [Online; accessed 30-June-2017].

2. Xiahai Zhuang and Juan Shen, "Multi-scale patch and multi-modality atlases for whole heart segmentation of mri," Medical image analysis, vol. 31, pp. 77-87, 2016.

3. X Zhuang, S Ourselin, R Razavi, DLG Hill, and DJ Hawkes, "Automatic whole heart segmentation based on atlas propagation with a priori anatomical information," Medical Image Understanding and Analysis-MIUA, pp. 29-33, 2008.

4. Peng Peng, Karim Lekadir, Ali Gooya, Ling Shao, Steffen E Petersen, and Alejandro F Frangi, "A review of heart chamber segmentation for structural and functional analysis using cardiac magnetic resonance imaging," Magma (New York, $N Y$ ), vol. 29, pp. 155, 2016.

5. Aliasghar Mortazi, Rashed Karim, Kawal Rhode, Jeremy Burt, and Ulas Bagci, "CardiacNet: Segmentation of left atrium and proximal pulmonary veins from mri using multi-view cnn," arXiv preprint arXiv:1705.06333, 2017.

6. Rudra PK Poudel, Pablo Lamata, and Giovanni Montana, "Recurrent fully convolutional neural networks for multi-slice mri cardiac segmentation," arXiv preprint arXiv:1608.03974, 2016.

7. MR Avendi, Arash Kheradvar, and Hamid Jafarkhani, "A combined deep-learning and deformable-model approach to fully automatic segmentation of the left ventricle in cardiac mri," Medical image analysis, vol. 30, pp. 108-119, 2016.

8. Gongning Luo, Ran An, Kuanquan Wang, Suyu Dong, and Henggui Zhang, "A deep learning network for right ventricle segmentation in short-axis mri," in Computing in Cardiology Conference (CinC), 2016. IEEE, 2016, pp. 485-488.

9. Alexandre de Brébisson and Pascal Vincent, "The Z-loss: a shift and scale invariant classification loss belonging to the spherical family," arXiv preprint arXiv:1604.08859, 2016.

10. Martín Abadi, Ashish Agarwal, Paul Barham, Eugene Brevdo, Zhifeng Chen, Craig Citro, Greg S Corrado, Andy Davis, Jeffrey Dean, Matthieu Devin, et al., "TensorFlow: Large-scale machine learning on heterogeneous distributed systems," arXiv preprint arXiv:1603.04467, 2016. 